\title{
Thermo-Chemical Analysis of the Cure Process of Thick Polymer Composite Structures for Industrial Applications
}

\author{
O.S. Dmitriev*, A.A. Zhyvenkova, A.O. Dmitriev \\ Department of Physics, Tambov State Technical University, \\ 112, Michurinskaya St., Tambov, 392032, Russian Federation \\ *Corresponding author. Tel.: + 7 (4752) 6303 69.E-mail:phys@nnn.tstu.ru
}

\begin{abstract}
This study contributes to the understanding of the thermo-chemical processes occurring in the cure of polymer composites, especially in thick structures. During the manufacturing process, thermo-chemical properties of the material undergo considerable changes and are affected by a high number of factors. Therefore, the main part of this analysis is the improvement of the strategy and the experimental study of the material properties behavior during the manufacturing process of the polymer composite. The thermo-chemical properties, simulation of the cure process and the calculation of the optimal production cycle of thick polymer composite structures are executed by a specialized computer-measuring system. The results of the study of the thermo-chemical properties during the curing of polymer composites and optimal cure cycles for industrial applications of thick structures manufacturing are presented.
\end{abstract}

\section{Keywords}

Manufacturing process; optimal cure cycles; polymer composites; simulation; thermo-chemical properties; thick structures.

(c) O. S. Dmitriev*, A.A. Zhyvenkova, A.O. Dmitriev, 2016

\section{Introduction}

The manufacturing process of polymer composites and their structures is a complex, energy-intensive and time-consuming. It is related to periodic chemical processes. Therefore, the task of raising the intensity of polymer composite structures production is mainly related to the performance of manufacturing operations for producing a polymer composite material, such as curing, heat treatment and cooling $[1,2]$.

The intensity of polymer composite products manufacture is directly related to their quality, which is determined by the properties of the cured composite, its micro and macro structure and the geometrical dimensions of the finished product. The structure of the product material is largely dependent on the processing parameters and determines the optimal manufacturing processes of heat and mass transfer in the curing step. The use of non-optimum temperaturetime cure cycle can lead to overheating of the inner layers of the product material, degradation of the matrix resin and associated porosity increase, the accumulation of residual stresses which decrease the strength properties of the material, the deviation degree of crosslinking of the polymer matrix from the optimum, the distortion and deviation from the desired finished product geometry, etc. To eliminate these shortcomings and to ensure low cost and high quality products, as well as to increase the intensity of the process it is necessary to use the optimal temperaturetime cure cycle [3-9]. Thus, the main tasks that must be solved in determining the optimal cure cycle of thick polymer composite structures are as follows:

- reduction in the duration of the cure cycle and energy consumption;

- decreasing of inhomogeneities in the temperature and conversion across the thickness of the composite;

- complete cure of resin in the final composite material;

- compaction of the composite up to the desired thickness of the product. 
Determining the optimal cure cycle of polymer composite products is a difficult and responsible task involving considerable challenges related to the lack of a theory describing the entire process of curing the material. Until recently, the empirical techniques were used to choose cure cycles. They are based on the laboratory studies of the dependency of various properties of the polymer composites on cure cycle, for example, tensile strength or bending on the temperature and the molding pressure, etc. In $[10,11]$ the curing cycle of products based on epoxy and epoxy-phenol-formaldehyde resin was determined by the gelation time studied using differential thermal analysis and differential scanning calorimetry. The main disadvantages of these techniques are high expenditures of labor, duration and non-optimality of obtained process cycles. They can not take into account possible overheating of the inner layers of the product owing to heat generation by the exothermic cure reaction, the mass transfer of resin under pressure and more. Therefore, the results obtained by using these techniques do not fully correspond to the processes taking place during the curing of materials in the actual structures. They can be generally applied to thin-walled plates (1-3 mm thick), which realize uniform curing of the material thickness, and can not be used for thick products (more than 3-5 mm), in which the entire course of the curing is determined by the speed dissipation of heat (by heat conductivity) released by the exothermic cure reaction. The curing cycles of thick products are usually prepared by the cure cycles of thin plates that are obtained empirically. In this case, the temperatures of the isothermal holds do not change, and the hold time in the mold is set as a linear dependence on the thickness of the product $[1,2]$. This technique does not take into account the heat transfer, the curing kinetics and the processes associated with changes in resin viscosity, which lead to large errors.

The modern methods of designing the optimal cure cycles of polymer composites are based on the use of mathematical models. They consist in the formulation and numerical solution of the extreme problem of minimizing some optimality criterion, ensuring the creation of high-quality finished product in minimal time with minimal residual stress [3-7]. By means of this concept it is possible to determine the cure cycles of the polymer composite products for any geometric sizes and shapes.

Process simulations for polymer composites curing use physically based models to simulate the process and predict the cure time and other properties of the final product. Simultaneously we are trying to solve two conflicting objectives. On the one hand, it is necessary to obtain a simple model with a small number of parameters and the research equipment is available for it. On the other hand, the model should be adequate to the real process with acceptable accuracy. The simple linear mathematical models excluding many process phenomena, such as leakage of the resin under pressure, the dependence of the thermophysical properties on temperature, degree of cure and content of the resin lead to large errors in simulation and unreliability of the solutions obtained. Moreover, we must bear in mind that a mathematical model for each specific technology has its own characteristics, but also a common basis, and must be chosen according to the method used for product formation $[1,2]$.

There are two governing equations used in the process model. One is the energy equation, and the other is a cure kinetics equation. Sometimes the rheological equation of the resin flow is added. These equations make up a transient coupled problem. Therefore, an important step of simulation is the properties analysis of polymer composites curing as the parameters of the mathematical model [12-14]. Therefore, the purpose of this work is to develop strategies and experimental study of the thermochemical properties of the material during polymer composite manufacturing which is necessary for simulation of the curing process and calculation of optimal production cycles of thick polymer composite structures.

\section{Experimental Strategy}

The main parameters of the mathematical model of heat and mass transfer process and chemical kinetics during the cure are thermo-chemical properties (TCP) of the material, which include: the thermophysical properties (TPP), i.e. the volume heat capacity $C$ and thermal conductivity $\lambda$ in cured $C_{\mathrm{cd}}, \lambda_{\mathrm{cd}}$ and in uncured $C_{\mathrm{pr}}, \lambda_{\mathrm{pr}}$ state and during the cure $C(T, \beta, \gamma), \lambda(T, \beta, \gamma)$ in the transition from the uncured to the cured state, the rate of evolved heat $W(t)$, full heat of cure reaction $Q_{\mathrm{f}}$, and also the kinetic properties, i.e. the activation energy of the cure process $E(\beta)$, kinetic function $\varphi(\beta)$, which includes the speed and the order of the chemical reaction, and if necessary, rheological properties, i.e. the activation energy of viscous flow during the cure $E_{\mu}$ and the effective viscosity of the resin $\widetilde{\mu}(\beta)$. These properties are not constants. They are functions of the temperature $T$, the degree of cure $\beta$, the resin content $\gamma$ in the material during the cure or time $t$, which should be determined from the experimental data. 
However, experimentally using a single test, we can only measure the temperature dependence of the thermophysical properties and by performing few tests of the TPP measurement with different ratios of ingredients we can also determine experimentally the dependence of the TPP on the resin content $\gamma$. Experimental determination of the TPP depending on the degree of resin cure $\beta$ is an extremely difficult task because of the temperature-time character of the current chemical curing reaction. Therefore, along with the experimental determination of the temperature dependence, the approximation methods should be used for TPP dependence on the degree of cure $\beta$ and the resin content $\gamma$.

Devices that are currently used in physical and chemical laboratories, such as IT-C-400, IT- $\lambda-400$, IT-4 (Russia), TC-3000H/L by "SINKU-RIKO" (Japan), Netzsch LFA 457 MicroFlash Thermal Analyzer (Germany) are generally intended for studies of the thermophysical properties of solid materials. To study the heat capacity and kinetics of polymer composites curing differential scanning calorimeters (DSC) or instruments for thermogravimetric and differential thermal analysis (DTA) were used, for example DSC 204 F1, DSC 404 F1 Phoenix, DSC 214 Polyma, STA 449 F3 Jupiter by "NETZSCH" (Germany) and others. They are usually intended for studies of small size samples in the absence of process pressure. Appliances intended to determine the rheological resin properties of polymer composites are based on the laws of classical rheology and allow measuring the properties of pure resin excluding the structural features of the composite and manufacturing process [15]. These factors limit the application of standard devices and methods for the curing of composites study, as they do not allow to fully determine all the parameters of the mathematical model of the cure process with all the features and interconnections. Thus, to solve this problem it is necessary to use special methods and instrumentation that will determine the properties of polymer composites near the process conditions of obtaining the composite products. Using this approach all the features of the polymer composites properties can be more accurately analyzed, and the parameters of the mathematical model corresponding to the real object can be obtained.

The thermophysical $C(T, \beta, \gamma), \lambda(T, \beta, \gamma), W(t), Q_{\mathrm{f}}$, the kinetic $E(\beta), \varphi(\beta)$ and the rheological $E_{\mu}, \widetilde{\mu}(\beta)$ properties of polymer composites, determined in the process conditions, are in some ways effective $C_{\mathrm{w}}, \lambda_{\mathrm{w}}$, i.e. they are relevant features of the process which include unaccounted factors and effects in simulations. Therefore, the promising methods and devices are those which allow us to study the materials of real structures with special samples and to implement the process conditions in the study such as process pressure, heating rate, especially laying prepreg and explore filled polymer composite instead of pure resin, etc. These requirements are explained by the presence of interfacial interactions at the boundary separating the resin-filler and the influence on the kinetics of cure, as well as the influence of process pressure and laying on the thermal conductivity of the polymer composite $[15,16]$.

For this reason, a specially designed computermeasuring system (CMS) has been used to study the parameters of the mathematical model of the polymer composites cure process [17]. CMS consists of the subsystems made for the research and calculation of optimal cure cycles (CAD). Subsystem of research is designed for controlling the experiment, for acquisition, processing and systematization of data, for the solution of inverse problems of heat conduction, kinetics and rheology, i.e. the subsystem allows determining the parameters of the mathematical model of the cure process. The diagram of the experiments, data acquisition and processing is shown in Fig. 1.

The hardware of the CMS subsystem to study the curing of polymer composites is designed on the PC. It includes: the measuring device, the unit preamplification of the signals of thermocouples, the controlled power supply unit for heaters and the measuring interface that represents adapter of analog and digital I/O installed into the PC. The measuring device, the unit amplification and the power supply unit are a special development of the research subsystems of CMS.

The measuring device creates the necessary conditions for heating and curing of the test samples, capable of measuring the temperature $T_{i, j} \equiv T(x, t)$ in $i=2-5$ points across the thickness of the sample and the heat flux $q_{\mathrm{j}} \equiv q(t)$ from the main heater. It is designed on the principle of adiabatic calorimeter with a system of electric heaters and compression rheometer, capable of creating and measuring the pressure $P(t)$ applied to the sample during heating and curing, and to measure its thickness $L(t)$ versus time. This allows in a single experiment combined with the thermophysical and kinetic properties to determine the rheological properties of the resin in the longitudinal direction of the flow. The construction of the measuring device allows studying samples in the temperature range from 20 to $270{ }^{\circ} \mathrm{C}$ under a pressure of $2 \mathrm{MPa}$. 


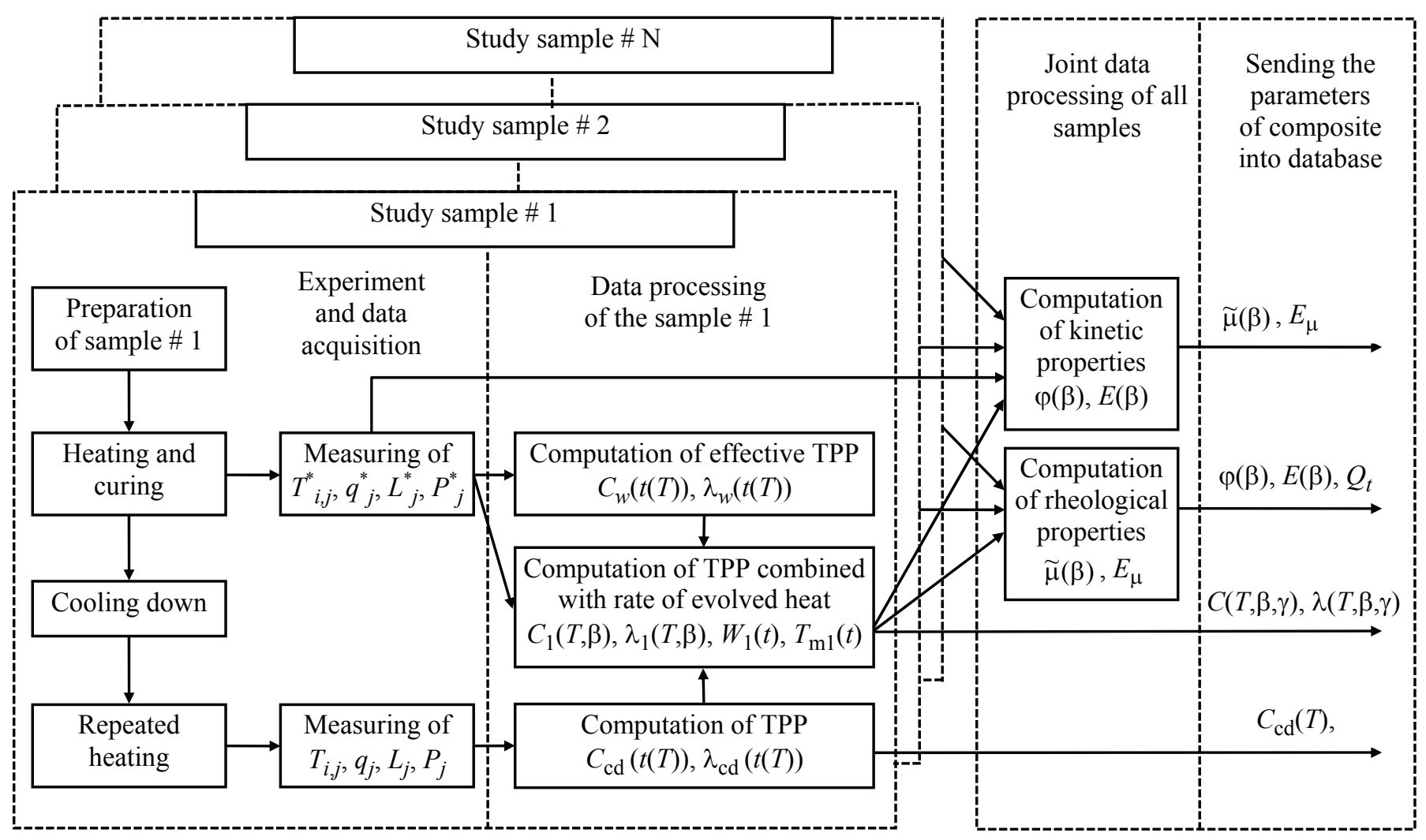

Fig. 1. The diagram of the experiments, data acquisition and processing

The main and three adiabatic heaters are stipulated in the measuring device in order to maintain adiabatic conditions and a special temperature mode of heating, as well as implementation in the test sample of unidirectional heat transfer. Measurement of temperature in the measuring device is also performed using the three measuring and two differential thermocouples embedded into the heaters substrates.

The software of the CMS to study the curing of polymer composite materials is developed on the basis of models and methods used for the study of thermophysical, kinetic and rheological properties of materials [14]. To calculate the thermophysical properties of $C(T, \beta, \gamma), \lambda(T, \beta, \gamma)$, we used the method of integral characteristics [6]. The kinetic properties $E(\beta)$ and $\varphi(\beta)$ were determined by the method of equal conversions, using the rate of evolved heat of cure reaction $W(t)$. The rheological properties $E_{\mu}$ and $\widetilde{\mu}(\beta)$ were also determined by the method of equal conversions, using thickness variation sample $L(t)$. The objectives mentioned above are the inverse problems of heat conduction, kinetics and rheology. Therefore, special techniques have been developed to address them.

Also, the software of the CMS includes a database and a number of supporting procedures: programs of numerical solution of nonlinear differential and integral equations, the program to support decision- making when choosing the method of calculating the thermophysical properties, the program of spline smoothing of experimental data, etc.

The database of the CMS is intended for storing the thermo-chemical properties of the studied composites and other parameters of the mathematical model. For easy search, the experimental information on the composites in the database is integrated into the generalized groups and subgroups on the basis of the similarity of their formulations, structure and properties. The database allows comparing the properties of composites when changing the content of the ingredients and tracing the dynamics of change. This in turn makes it possible to predict the properties in the absence of material data on available information in the database.

Using the experimental data stored in the database, the calculating subsystem of the CMS allows performing simulations and analysis of temperatureconversion fields, representing them in the form of graphs and 3D surfaces, analyzing irregularity of the curing, quick

ly assessing the advantages and disadvantages of the cure cycle used for the developed material and choosing the right strategy of the cycle optimization. At last the CMS software can implement the calculation of the optimal processing cycles of polymer composite products for any geometric sizes and shapes on the basis of hot curing prepregs. 


\section{Results and Discussion}

Experimental study of the thermo-chemical properties of polymer composites during the curing consists in heating and curing the test sample in the measuring device of CMS. Special mode of heating the test sample in the measuring device is implemented using CMS hardware and software.

The studied sample is a package in the form of a square with the sides of $100 \mathrm{~mm}$, made up of several layers of prepreg studied material with a thickness of $5 . . .20 \mathrm{~mm}$. Stacking layers of prepregs and orientation fibers of the sample are made similarly to industrial products: unidirectional, cross or diagonal-cross, etc. The samples of materials prepared in this way and their sizes allow to take into account, while studying effective properties, the rheological processes that occur during the curing, consider the details of prepreg stacking layers in the product, average a local inhomogeneity of the material structure and the factor content of resin that does not allow to perform other standard methods and devices, including the method of differential scanning calorimetry.

The study of each sample is performed in two stages: heating together with curing and re-heating after cooling the cured sample (Fig. 1). During the first stage we obtain an experimental thermogram for studying the kinetics of cure and resin flow, as well as determining the effective heat capacity $C_{w}(T)$, including the rate of evolved heat of cure reaction $W(t)$, and the effective thermal conductivity $\lambda_{w}(T)$. At the second stage we obtain the thermogram of the re-heating by which we define the thermophysical properties of the cured material $C_{\mathrm{cd}}(T), \lambda_{\mathrm{cd}}(T)$, necessary for calculating the TPP depending on the degree of cure $\beta$ and simulation of the curing process. Repeated experiments were done for each heating rates and for several samples.

Using software of CMS by the experimental data of monotonic heating and curing of several samples of the studied material there was first calculated the temperature dependence of TPP cured material $C_{\mathrm{cd}}(T), \lambda_{\mathrm{cd}}(T)$ and then - the effective TPP of $C_{w}(T)$, $\lambda_{w}(T)$. Based on them there were calculated by iterations the rate of evolved heat of cure reaction $W(t)$, the full heat of cure reaction $Q_{\mathrm{f}}$ and TPP during curing $C(T, \beta)$ and $\lambda(T, \beta)$ depending on temperature $T$ and degree of cure $\beta$, the corresponding factor content of the resin in the prepreg $\gamma$. The overall heat evolved in the reaction was determined as the average value of reaction heats calculated from each experiment $[6,17]$.

The kinetic properties $E(\beta)$ and $\varphi(\beta)$ were determined by the rate of evolved heat of cure reaction $W(t)$, measured in dynamic scans of the curing of two or more samples at different heating rates $T_{\mathrm{m}}(t)$. The rheological properties $E_{\mu}$ and $\widetilde{\mu}(\beta)$ were determined by measuring the thickness $L(t)$ of curing sample at squeezing-out of resin under pressure with the change in viscosity.

The results of experimental studies of thermophysical and kinetic properties in the cure process of CMU-7 carbon fiber-reinforced plastic samples with EDT-69N epoxy polymer matrix are shown in Fig. 2-4.

The graphs show (Fig. 2) that the initial part of the effective thermophysical properties $C_{w}(T), \quad \lambda_{w}(T)$ before the beginning of intensive curing reaction characterizes the properties of uncured prepreg $C_{\mathrm{pr}}(T)$, $\lambda_{\text {pr }}(T)$. The beginning of intensive curing reaction (in Fig. 2 is a $110-120^{\circ} \mathrm{C}$ ) corresponds to a rapid decrease in the effective volume heat capacity $C_{w}(T)$, because it includes the rate of evolved heat of cure reaction $W(t)$ and it is in this part of the effective exothermic heat capacity. Effective thermophysical properties $C_{w}(T), \lambda_{w}(T)$ at the final stage of the experiment at a high degree of cure $\beta$ merge with TPP cured material $C_{\mathrm{cd}}(T), \lambda_{\mathrm{cd}}(T)$, which corresponds to the transition of polymer composite in the cured state. As seen from Fig. 2 the dependence of the thermophysical properties of cured composite $C_{\mathrm{cd}}(T)$, $\lambda_{\mathrm{cd}}(T)$ and uncured prepreg $C_{\mathrm{pr}}(T), \quad \lambda_{\mathrm{pr}}(T)$ are equidistant curves. This allows for the difference between the TPP cured composite and uncured prepreg

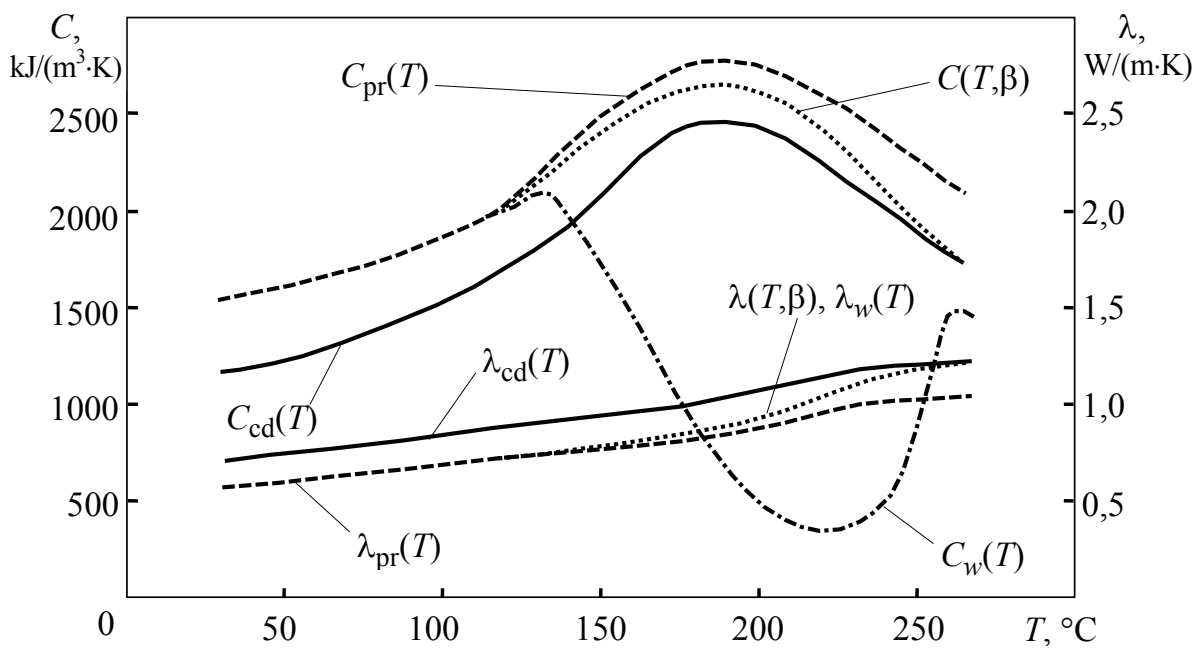

Fig. 2. Thermophysical properties of $\mathrm{CMU}-7$ carbon plastic 


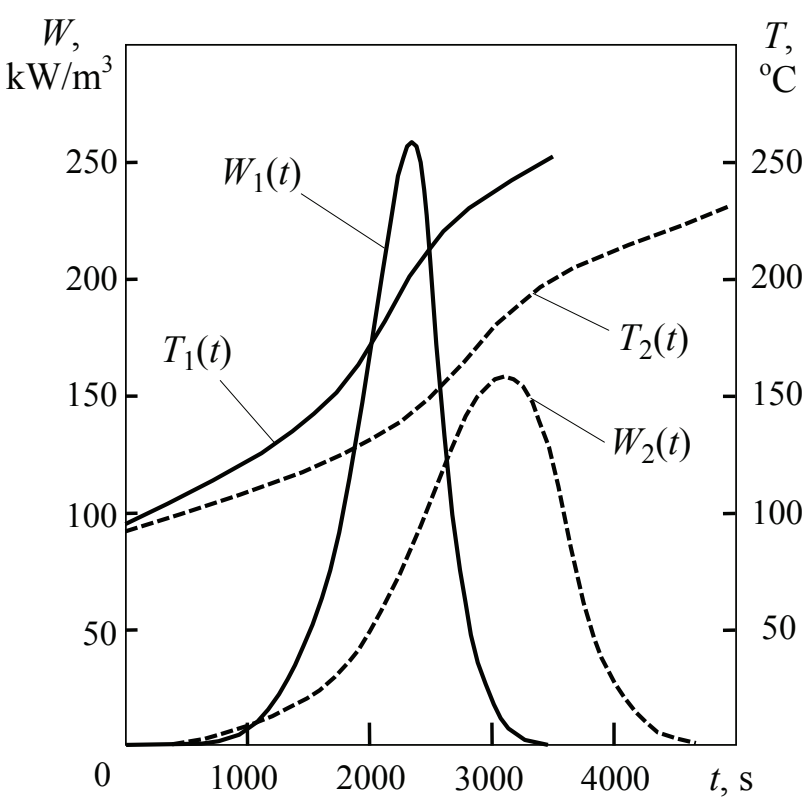

Fig. 3. Evolved heat of cure reaction of CMU-7 carbon plastic

$\Delta C$ and $\Delta \lambda$ at the time of the beginning of the curing reaction, used for the calculation of TPP in the cure process of $C(T, \beta), \lambda(T, \beta)$. They are determined by the TPP of prepreg $C_{\mathrm{pr}}(T), \lambda_{\mathrm{pr}}(T)$ in the range from room temperature to the temperature at the beginning of the curing reaction, and above the temperature of the beginning of the curing reaction it is determined by $C_{\mathrm{cd}}(T), \lambda_{\mathrm{cd}}(T)$ and $\Delta C, \Delta \lambda$.

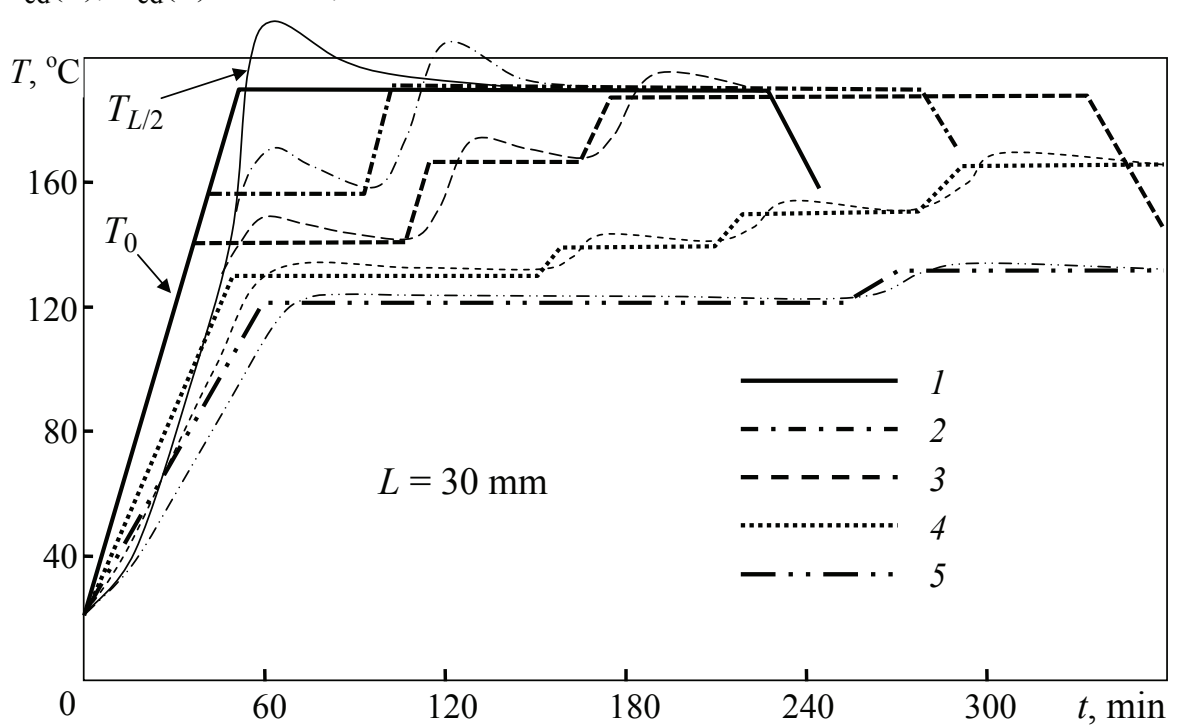

Fig. 5. Simulated temperature cure profiles of carbon plastic for a $30 \mathrm{~mm}$ thick product, calculated for various constraints: 1 - no constraints, the simulated temperature overshoot $\theta=21 \mathrm{~K}$, the simulated temperature gradient $\chi=2,9 \mathrm{~K} / \mathrm{mm}$;

2 - when specifying constraints $\bar{\theta}=15 \mathrm{~K}, \bar{\chi}=2 \mathrm{~K} / \mathrm{mm}$;

3 - when specifying constraints $\bar{\theta}=10 \mathrm{~K}, \bar{\chi}=1 \mathrm{~K} / \mathrm{mm}$;

4 - when specifying constraints $\bar{\theta}=5 \mathrm{~K}, \bar{\chi}=0,4 \mathrm{~K} / \mathrm{mm}$;

5 - optimal cure cycle, when specifying constraints $\bar{\theta}=5 \mathrm{~K}, \bar{\chi}=0,2 \mathrm{~K} / \mathrm{mm}$

(Note that only two steps of the cycle are shown in Fig. 6)

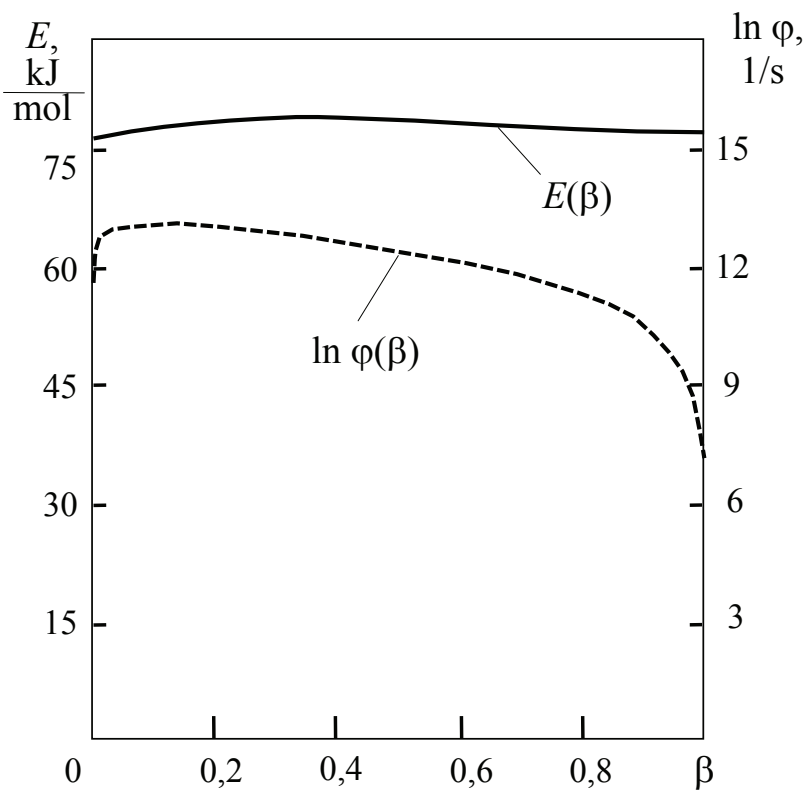

Fig. 4. Kinetic properties of CMU-7 carbon plastic

The rate of evolved heat of cure reaction $W(t)$ (Fig. 3) has a pronounced extremum, corresponding to the transition from a liquid resin to the viscous-flow state (gel point). With the increase in the rate of heating the extreme of evolved heat of cure reaction $W(t)$ shifts towards higher temperatures. At the same time, the duration of heat generation and gelation time with increasing heating rate decreases. The area under the curve of evolved heat of cure reaction $W(t)$ characterizes the full thermal effect $Q_{\mathrm{f}}$ of curing reaction of the resin, and the ratio of the thermal effect $Q$ to the full heat of cure reaction of $Q_{\mathrm{f}}$ corresponds to the degree of completeness of the curing process or calorimetric degree of cure $\beta$. The full thermal effect of the curing reaction of CMU-7 carbon fiber-reinforced plastic with a mass fraction of the resin in the prepreg $\gamma_{\mathrm{s}}=53 \%$ of $Q_{\text {fcom }}=1,99 \cdot 10^{8} \mathrm{~J} / \mathrm{m}^{3}$. In terms of unit mass of pure resin the full thermal effect corresponds to $Q_{\text {frs }}=3,75 \cdot 10^{8} \mathrm{~J} / \mathrm{m}^{3}$.

The kinetic properties, i.e. the activation energy of the cure process $E(\beta)$ and kinetic function $\varphi(\beta)$ calculated by the rates of evolved heat of cure reaction $W_{1}(t)$, $W_{2}(t)$ during the curing of two samples at different heating 
rates $T_{1}(t), T_{2}(t)$ are shown in Fig. 4. The above mentioned parameters are functions of the degree of cure $\beta$ and express the multiple stages of the curing process. The kinetic function $\varphi(\beta)$ is represented in the figure in semi-logarithmic scale because of its significant change during cure.

Calculation subsystem of CMS also has the option of numerical simulation of the temperatureconversion fields for analysis. It verifies the various strategies in order to optimize the cure cycles of polymer composites, clarify optimality criteria $I$ and determine the values of the constraints imposed on the cure process. The influence of the set point value of the temperature overshoot $\bar{\theta}$ and the value of the temperature gradient $\bar{\chi}$ on the simulated cure cycle is studied. The simulation analysis of the cure cycles of carbon plastic CMU-7 for a $30 \mathrm{~mm}$ thick product, which have been calculated for various constraints $\bar{\theta}$ and $\bar{\chi}$, are shown in Fig. 5 as the temperature profiles on the surfaces $T_{0}$ and in the middle $T_{L / 2}$ of the plate. In the one-step cure cycle with isothermal hold $190{ }^{\circ} \mathrm{C}$ the maximum temperature inside the flat product with thickness $L=30 \mathrm{~mm}$ reaches $T_{L / 2}=211^{\circ} \mathrm{C}(\theta=21 \mathrm{~K})$, and the temperature gradient reaches $\chi=2,9 \mathrm{~K} / \mathrm{mm}$, which leads to the destruction of the resin and the formation of the strained material structure. The cure cycle becomes multistep and longer at imposing more stringent constraints (the temperature cure profiles $2,3,4,5$ ).

The first step in solving the optimization problem and calculating the optimal cure cycle is the selection optimality criterion of the manufacturing process $I$ $[4,5,9]$. The objective function of the optimization problem may consist in minimizing the cycle time of the cure process $t_{\mathrm{f}}$, minimizing energy consumption $Q^{*}$ or minimizing residual stresses $\sigma$. The mathematical formulation of the optimization problem of the cure cycle is to solve the equations of the mathematical model corresponding to the current technique of manufacture polymer composites and finding the temperature-time cycle $U$ on the surfaces of the heating and curing product, which minimizes the chosen optimality criterion $I$ and complies with the constraints imposed on the process considering the temperature-time cycles allowable for equipment. The necessary temperature-time cure cycle is calculated as a piecewise linear function. A condition for the finish of solving the optimization problem for the cure cycle is achieving a given finite degree of cure throughout the volume of the product. As a result of the calculation, we obtain the optimal cure cycles, which guarantees high-quality finished products [4-6]. The results of optimization cycles for curing flat products with various thicknesses of carbon plastic CMU-7 are shown in Fig. 6.

The analysis of the temperature profiles (Fig. 6) shows that the duration of the cure process increases significantly with increasing thickness of the polymer composite product, and the temperature of the first and subsequent isothermal holds decreases significantly, thus the internal heat release and temperature of the inner layers on the first stages of the process are also reduced. This reduces to a predetermined level the heterogeneity of the temperature and conversion field and leads to increasing the product quality which allows to use the calculated optimal cure cycles for the production of large-sized thick-walled polymer composite products.

\section{Conclusion}

The present work analyzes thermo-chemical properties of the material that are intended to be used for the production of a thick composite object. It is shown that during the manufacturing process thermochemical properties of the material vary considerably. The thermophysical properties of the material were studied in different states: cured state, uncured state, upon curing, during the transition from the uncured 
to the cured state. The kinetic properties of the curing process were calculated by the rate of evolved heat upon curing of two samples at different heating rates. The thermophysical and kinetic properties upon curing were studied in order to obtain parameters for the simulation and optimization of the curing cycles. This work shows that the experimental study of thermo-chemical properties of the materials and optimal curing conditions, though it may sound costly and time-consuming, might help identify unforeseen critical situations and avoid the lack of performance in the final thick composite structures.

\section{References}

1. Kerber M. L., Vinogradov V. M., Golovkin G. S., etc., (2008). Polymer composites: Structure, properties and technology, Ed. A.A. Berlin. S. Petesburg, Professia. (Rus)

2. Hollaway L. C. (1990). Polymers and Polymer Composites in Construction. London, T. Telford.

3. Jahromi P.E., Shojaei A., Reza Pishvaie S.M. (2012). Prediction and optimization of cure cycle of thick fiber-reinforced composite parts using dynamic artificial neural networks. Journal of Reinforced Plastics and Composites, 31, 18, 1201-1215.

4. Dmitriev O.S., Mischenko S.V., Dmitriev S.O., Kirillov, V. N. (2009). Optimization of curing cycles products of the polymer composite materials on base of glutinous prepregs. Polymer Science. Series D, 2, 4, 223-229.

5. Dmitriev O., Mischenko S. (2011). Optimization of Curing Cycles for Thick-wall Products of the Polymeric Composite Materials. In book: Advances in Composite Materials - Ecodesign and Analysis, Edited by Brahim Attaf. InTech, Ch. 7, 141-160.

6. Dimitriev O.S., Mischenko S.V., Shapovalov A.V. (1997). Choice of curing cycles of vulcanizing rubber composites. KGK Kautschuk Gummi Kunststoffe, 50, 2, 98-101.

7. Brauner C., Soprano P., Herrmann A.S., Meiners D. (2015). Cure-dependent thermo-chemical modelling and analysis of the manufacturing process of an aircraft composite frame. Journal of Composite Materials, 49, 8, 921-938

8. Vafayan M., Beheshty M.H., Abedini H., Ghreishy, M.H.R. (2013). Effect of cure kinetic simulation model on optimized thermal cure cycle for thin-sectioned composite parts. Polymer Composites, 34, 7, 1172-1179.

9. Ruiz E., Trochu F. (2006). Multi-criteria thermal optimization in liquid composite molding to reduce processing stresses and cycle time. Composites Part A: Applied Science and Manufacturing, 37, 6, 913-924.

10. Khairullin I.I., Aleksashin V.M., Petrova A.P. (2007). Use of thermal analysis techniques for studying a hot-melt adhesive // Polymer Science Series C, 49, 1, 84-88.

11. Stern W.E. (1992). A Practical Method for the Cure Cycle Optimization of Epoxy Resins Via Differential Scanning Calorimetry. 37th International SAMPE Symposium. 37, March 9-12. 231-239.

12. Brauner C., Block T.B., Purol H., Herrmann A.S. (2012). Microlevel manufacturing process simulation of carbon fiber/epoxy composites to analyze the effect of chemical and thermal induced residual stresses. Journal of Composite Materials, 46, 17, 2123-2143.

13. Giorgini L., Mazzocchetti L., Benelli T., Minak G., Poodts E., Dolcini E. (2013) Kinetics and modeling of curing behavior for two different prepregs based on the same epoxy precursor: A case study for the industrial design of thick composites. Polymer Composites, 34, 9, 1506-1514.

14. Dmitriev O.S., Mishchenko S.V., Ponomarev S.V. (1998). Mathematical modeling of the curing process of polymer composite materials by hot pressing. Vestnik TGTU, 4, 4, 390-399. (Rus)

15. Lipatov Y.S. (1991). Physico-chemical bases filling polymers. Moscow, Khimiya. (Rus)

16. Mishchenko S.V., Dmitriev O.S., Shapovalov A.V. (1993). An automated system for investigation and selection of the optimum conditions of curing of thin-walled composite parts. Chemical and Petroleum Engineering, 29, 3, 144-148.

17. Dmitriev O.S., Mishchenko S.V., Dmitriev A.O., Kirillov V.N. (2010). Computer-Measuring System for Research into Properties of Glutinous Prepregs and Calculation of Curing Cycles of the Polymer Composite Materials on Their Base. Polymer Science, Series D, 3, 1, 20-25. 